\title{
Gas spectroscopy using low threshold mid-infrared radiation generated in $\mathrm{Si}_{3} \mathrm{~N}_{4}$ waveguides
}

\author{
Eirini Tagkoudi ${ }^{1}$, Davide Grassani ${ }^{1}$, Fan Yang ${ }^{2}$, Hairun Guo ${ }^{3}$, Tobias Kippenberg ${ }^{3}$, Camille-Sophie Brès ${ }^{1}$ \\ ${ }^{I}$ Ecole Polytechnique Fédérale de Lausanne (EPFL), Photonic Systems Laboratory (PHOSL), CH-1015, Switzerland \\ ${ }^{2}$ Ecole Polytechnique Fédérale de Lausanne (EPFL), Group For Fibre Optics (GFO), CH-1015, Switzerland \\ ${ }^{3}$ Ecole Polytechnique Fédérale de Lausanne (EPFL), Laboratory for Photonics and Quantum Measurements (LPQM), CH-1015, Switzerland \\ Author e-mail address: eirini.tagkoudi@epfl.ch
}

\begin{abstract}
We report trace-gas absorption spectroscopy based on the efficient generation of a 3.05 $\mu \mathrm{m}$ dispersive wave in a $\mathrm{Si}_{3} \mathrm{~N}_{4}$ waveguide pumped by a $2.09 \mu \mathrm{m}$ femtosecond mode-locked fiber laser.

OCIS codes: Nonlinear optics-Integrated optics (190.4390), Supercontinuum generation (320.6629), Absorption spectroscopy (300.1030).
\end{abstract}

\section{Introduction}

The middle infrared (Mid-IR) spectral region, where strong vibrational bands of molecular gases exist, has attracted intense interest for spectroscopy and sensing applications [1]. Mid-IR radiation can be generated through several techniques such as optical parametric oscillation (OPO) and difference frequency generation (DFG) [2], however wavelength conversion in nonlinear waveguides pumped by fiber lasers appears to be a promising and convenient solution for Mid-IR spectral coverage. Direct generation of Mid-IR light through dispersive wave generation $(\mathrm{DWG})$ in silicon nitride $\left(\mathrm{Si}_{3} \mathrm{~N}_{4}\right)$ waveguides, pumped with an erbium-doped fiber laser at $1.55 \mu \mathrm{m}$, established a significant first step towards compact frequency comb sources. However limitations in terms of conversion efficiency (CE) beyond $3 \mu \mathrm{m}$ implies input average power levels of hundreds of milliwatts to achieve the $\mathrm{mW}$-level Mid-IR power range required for fast spectroscopic application [3]. We recently proved that CE can be improved by optimizing the pump wavelength while maintaining the desired phase-matching conditions [4]. However, such scheme has never been used for gas sensing experiments yet.

Herein, we experimentally demonstrate absorption spectroscopy based on the on-chip efficient Mid-IR dispersive wave (DW) generated using a commercial tunable fs-fiber mode-locked laser (MLL) at $2.09 \mu \mathrm{m}$. We are able to achieve conversion efficiency up to the record value of $35 \%$ at $3.05 \mu \mathrm{m}$, meaning almost $5 \mathrm{~mW}$ of on-chip Mid-IR average power with only $13 \mathrm{~mW}$ of coupled pump power, by optimizing the pump wavelength and waveguide dispersion. We use this broadband Mid-IR source for direct absorption spectroscopy of acetylene $\left(\mathrm{C}_{2} \mathrm{H}_{2}\right)$.

\section{Experimental implementation and efficiency of DW}

The experimental setup is presented in Fig. 1(a). The pump is a commercial soliton self-frequency shifted thuliumdoped fiber MLL (Brevity $\lambda+$, NOVAE), with tunability from 2.04 to $2.09 \mu \mathrm{m}$, pulse duration (FWHM) of $\sim 78 \mathrm{fs}$ and repetition rate of $19 \mathrm{MHz}$. For the spectroscopy experiment, light from the output of the waveguide is directly coupled into a $1.09 \mathrm{~m}$ gas cell which contains the sample $\mathrm{C}_{2} \mathrm{H}_{2}$. At the cell's output, the collimated light is focused using a parabolic mirror into a fluoride fiber (ZBLAN fiber) and the spectra are recorded with an Optical Spectrum Analyzer (OSA). The $5 \mathrm{~mm}$ long straight $\mathrm{Si}_{3} \mathrm{~N}_{4}$ waveguide with a width of $1000 \mathrm{~nm}$ and height of $2090 \mathrm{~nm}$ is designed to produce anomalous dispersion at the pump wavelength and an expected phase-matched DW at $3 \mu \mathrm{m}$. The total losses are estimated as $\sim 12 \mathrm{~dB}$ for the TM fundamental mode while propagation losses are $0.2 \mathrm{~dB} / \mathrm{cm}$.
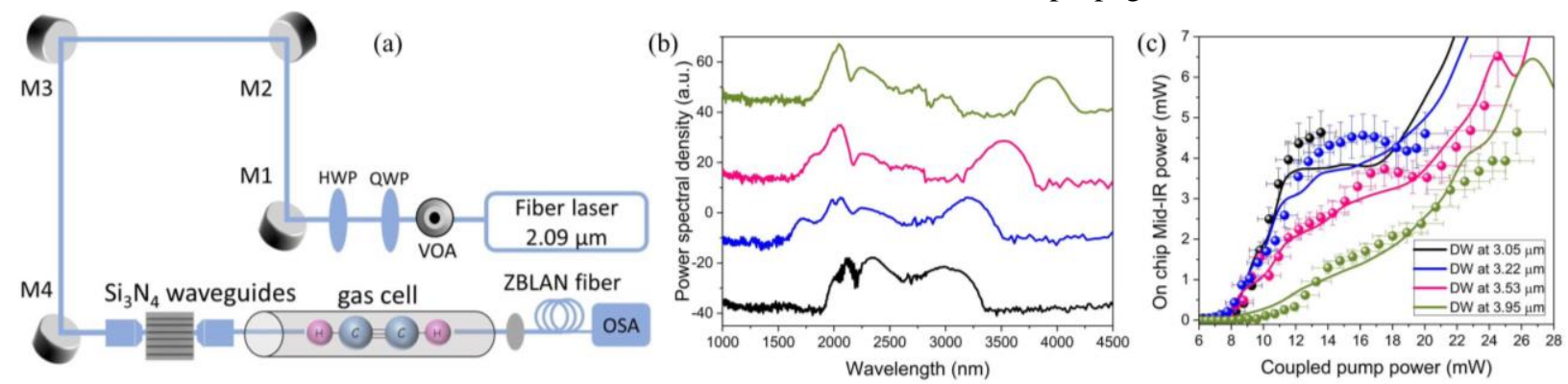

Fig.1 (a) Experimental setup. HWP: half wave plate, QWP: quarter wave plate, VOA: variable optical attenuator, M: mirror, OSA: optical spectrum analyzer (b) Experimental spectra of the $1000 \mathrm{~nm}, 1050 \mathrm{~nm}, 1100 \mathrm{~nm}$ and $1175 \mathrm{~nm}$ waveguide width (from bottom to top) for $13 \mathrm{~mW}$ average coupled power. (c) On-chip Mid-IR DW power as a function of coupled pump power for the different waveguides with DWs at $3.05 \mu \mathrm{m}$, $3.22 \mu \mathrm{m}, 3.53 \mu \mathrm{m}$ and $3.95 \mu \mathrm{m}$. (same color as in (b)). Points are experimental data, lines are simulations. Error bars indicate the uncertainty on DW power and coupled pump power. 
Mid-IR wavelength conversion is achieved through soliton-induced DWG within the supercontinuum process in the $\mathrm{Si}_{3} \mathrm{~N}_{4}$ waveguides. As clearly seen in the spectra of Fig. 1(b), for $13 \mathrm{~mW}$ of average coupled power we generate Mid-IR DWs in the 3-4 $\mu \mathrm{m}$ range by just changing the waveguide width, although with different efficiencies. The on-chip DW power is calculated considering coupling losses through the output ZBLAN fiber and the second chalcogenide lens after power calibration. In Fig 1(c) it can be seen that on-chip DW power of more than $4 \mathrm{~mW}$ can be obtained up to $4 \mu \mathrm{m}$ emission wavelength. Experimental results and simulations are in excellent agreement.

\section{Results}

The DW centered at $3.05 \mu \mathrm{m}$ overlaps with $\mathrm{C}_{2} \mathrm{H}_{2}$ absorption lines, a gas we had currently available, and we used it for the Mid-IR absorption spectroscopy experiment. Given the measured performances (Fig.1c), similar testing could be done up to at least $4 \mu \mathrm{m}$ with other gases. The transmitted spectra measured after the cell with and without the sample gas are presented in Fig. 2 (a). Characteristic absorption dips originating from $\mathrm{C}_{2} \mathrm{H}_{2}$ in the gas cell and from water vapors $\left(\mathrm{H}_{2} \mathrm{O}\right)$ outside the cell are clearly observed. The absorption spectrum for $\mathrm{C}_{2} \mathrm{H}_{2}$ in the cell is plotted by normalizing the sample spectrum $\left(396 \mathrm{ppm} \mathrm{C}_{2} \mathrm{H}_{2}\right.$ buffered in nitrogen $\left(\mathrm{N}_{2}\right)$ in total pressure 1 atm at $\mathrm{T}=296 \mathrm{~K}$ ) to the spectrum of pure $\mathrm{N}_{2}$ reference gas inside the long cell (Fig.2 (b)). The measurement time was approximately 2 minutes and the experimental results were compared with simulations based on HITRAN database. The maximum absorbance for $396 \mathrm{ppm} \mathrm{C}_{2} \mathrm{H}_{2}$ was measured as $8.8 \times 10^{-3} \mathrm{~cm}^{-1}$ while the standard deviation of residual absorbance for this global fit is $2.5 \times 10^{-4} \mathrm{~cm}^{-1}$. The signal-to-noise ratio (SNR) was calculated to be 35 and the noise equivalent concentration is $11 \mathrm{ppm}$. The differences between experimental data and simulations are given as residuals in Fig. 2(c). Spikes near the absorption peaks can be due to the limited resolution of the OSA (0.1 nm).

(a)

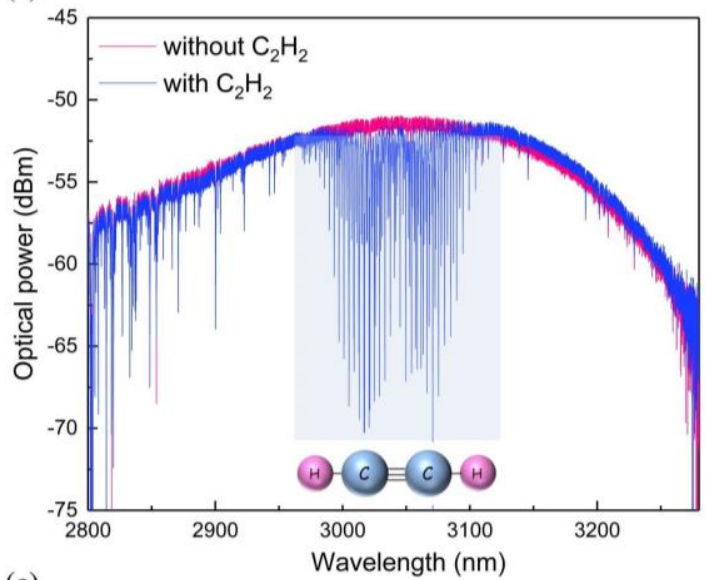

(6)

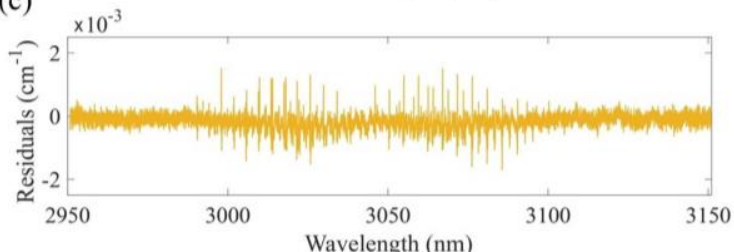

(b)

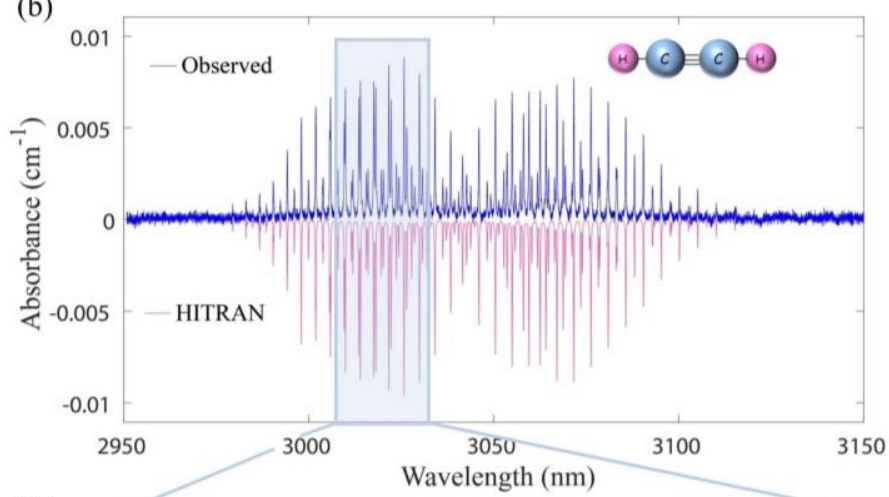

(d)

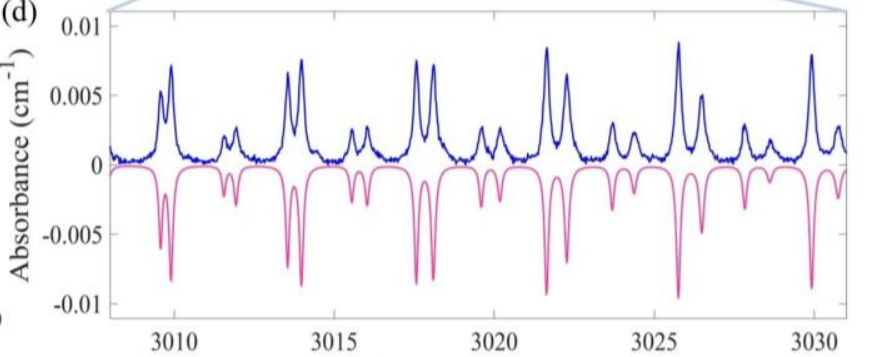

Fig. 2 (a) Transmitted spectrum of the empty (pink line) and filled (blue line) gas cell with $\mathrm{C}_{2} \mathrm{H}_{2}$ in $\mathrm{N}_{2}$ buffer gas at 1 atm total pressure. (b) Measured absorbance (blue) and theoretical absorbance obtained by HITRAN (pink). (c) Differences between simulations and experimental data of the multiple-line fit. (d) Zoom-in on the observed and HITRAN absorbance for the transmission window 3.01-3.03 $\mu$ m.

In summary, we demonstrate Mid-IR detection of $\mathrm{C}_{2} \mathrm{H}_{2}$ gas using a $3.05 \mu \mathrm{m}$ on-chip source generated from a fiber laser in a $\mathrm{Si}_{3} \mathrm{~N}_{4}$ waveguide. Out-of-the-chip $\mathrm{mW}$ power level can be obtained with about $13 \mathrm{~mW}$ of coupled pump power, and with just $26 \mathrm{~mW}$ at $4 \mu \mathrm{m}$, increasing by almost 1 order of magnitude the efficiency with respect to state of the art DFG in PPLN [2]. With further experiments we can envision on-chip multi-species detection in a chipsize CMOS compatible platform directly pumped with a commercial fiber MLL.

Acknowledgements: This work was supported by the European Research Council under the grant agreement ERC-MATISSE and ERC-PISSARRO.

[1] Vainio, M . et. al. 'Mid-infrared optical parametric oscillators and frequency combs for molecular spectroscopy', Physical Chemistry Chemical Physics, 18(6), 4266-4294, (2016).

[2] G. Ycas, et al., 'High-coherence mid-infrared dual-comb spectroscopy spanning 2.6 to $5.2 \mu \mathrm{m}$ ', Nat. Photonics 12, 202-208 (2018).

[3] Guo, H. et. al. 'Mid-infrared frequency comb via coherent dispersive wave generation in silicon nitride nanophotonic waveguides', Nature Photonics, 12(6), 330, (2018).

[4] Tagkoudi, E. et. al. 'Efficient mid-infrared dispersive wave generation in dispersion-engineered $\mathrm{Si}_{3} \mathrm{~N}_{4}$ waveguides pumped at $2 \mu \mathrm{m}$ '. In CLEO: Science and Innovations (pp. SW3A-8). OSA, (2018) 